
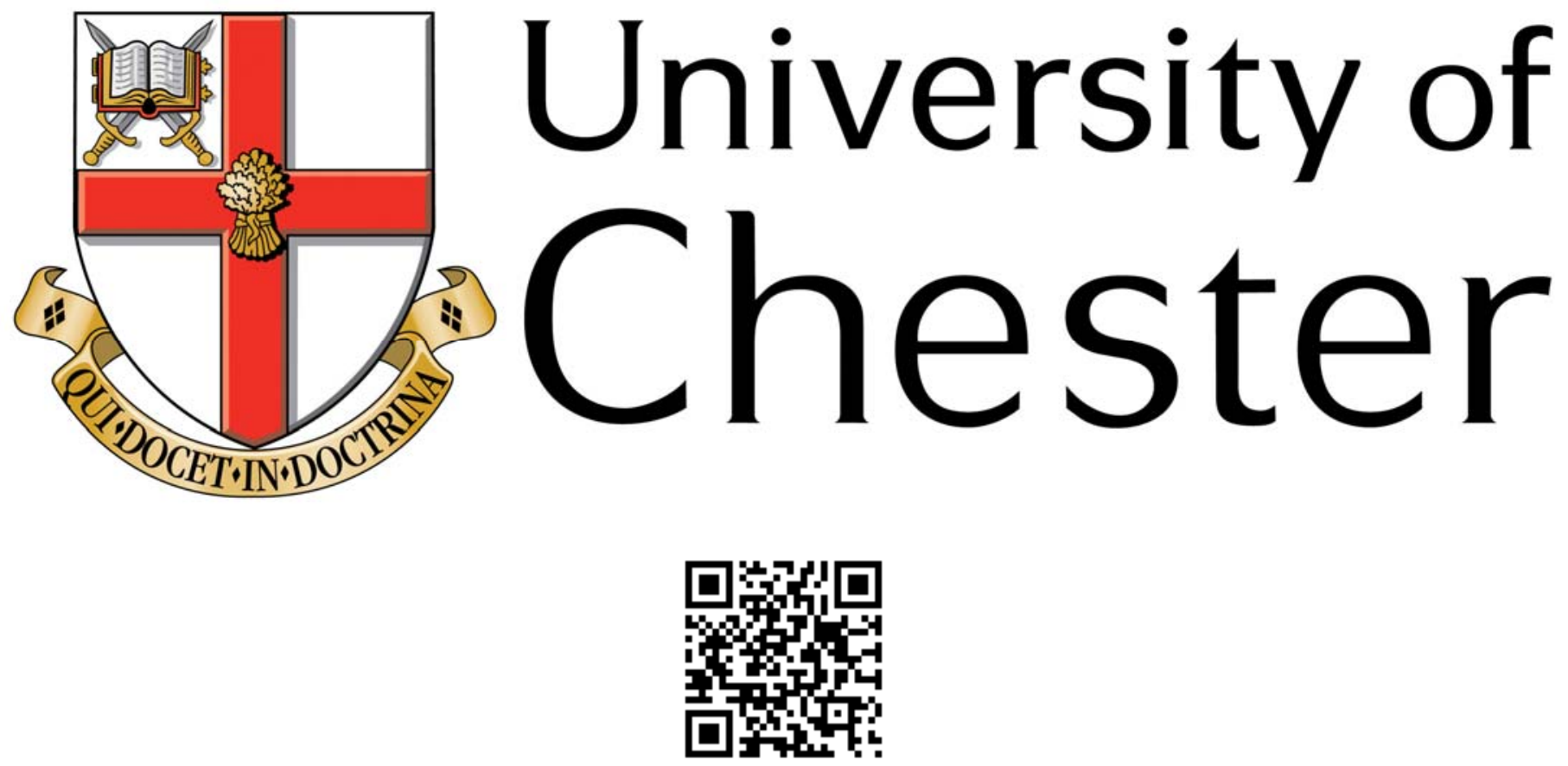

This work has been submitted to ChesterRep - the University of Chester's online research repository

\title{
http://chesterrep.openrepository.com
}

Author(s): Bushra Qayyum ; Mohammed Saeed ; Jason A Roberts

Title: Data aggregation in wireless sensor networks with minimum delay and minimum use of energy: A comparative study

Date: 9-10 March 2014

Originally published in: Proceedings of the BCS International IT Conference 2014, Abu Dhabi, United Arab Emirates, 9 - 10 March 2014

Example citation: Qayyum, B., Saeed, M., \& Roberts, J. A. (2014, March, 9-10). Data aggregation in wireless sensor networks with minimum delay and minimum use of energy: A comparative study. Proceedings of the BCS International IT Conference 2014, Abu Dhabi, United Arab Emirates, 9-10 March 2014. http://dx.doi.org/10.14236/ewic/bcsme2014.2

Version of item: Authors' accepted manuscript 


\section{Data Aggregation in Wireless Sensor Networks with Minimum Delay and Minimum Use of Energy: A comparative \\ Study}

Bushra Qayyum
PhD Student

Senior Lecturer

University of Chester
Dean of Research
Jason Roberts

University of Chester

\section{0@chester.ac.uk mohammed.saeed@khwarizmi.com_J.roberts@chester.ac.uk}

Abstract - the prime objective of deploying large- scale wireless sensor networks is to collect information from to control systems associated with these networks. Wireless sensor networks are widely used in application domains such as security and inspection, environmental monitoring, warfare, and other situations especially where immediate responses are required such as disasters and medical emergency. Whenever there is a growth there are challenges and to cope with these challenges strategies and solutions must be developed. This paper discusses the recently addressed issues of data aggregation through presenting a comparative study of different research work done on minimizing delay in different structures of wireless sensor networks. Finally we introduce our proposed method to minimize both delay and power consumption using a tree based clustering scheme with partial data aggregation.

\section{Keywords—Wireless sensor networks; Data aggregation; Delay; Energy consumption;}

\section{Introduction}

Wireless sensor networks, primarily used for disasters detection and, in best cases, prevention consist of autonomous sensors spatially distributed. A sensor may be grouped with other sensors to make a node. These nodes collect and cooperatively pass data to a management base or center which usually a powerful computer managing a huge database. Sensors in nodes monitor physical and/or environmental conditions such as images, sound, temperature, pressure, etc. Each wireless sensor network consists from few to hundreds or thousands of nodes depending on the project and each node mainly consists of a microcontroller, an interface circuit, a radio transceiver with antenna, and an energy source.

There is a revolutionary and ongoing research in this area and in particular on data aggregation and the reliability and efficiency of data transmission process from sources to destination(s).

Data aggregation in wireless sensor networks is the amalgamation of data coming from different sources while eliminating redundancy and thus minimizing the number of transmissions in order to save energy using datacentric networking rather than Address-centric networking (Krishnamachari et al 2002).

A major challenge for effective data aggregation is the management of energy consumption and delay. Most of the applications of wireless sensor networks where immediate response is crucial, such as disaster response, require minimum delays something that cannot be effectively achieved when measures for energy saving are implemented. The purpose for this research paper is to present a comparative study of research work done by examining these two important factors of data aggregation. The work will examine different event based data aggregation in different types of wireless sensor networks like cluster- and tree- based sensor networks. We will also discuss the process of partial aggregation and how to implement it with these parameters for future research work.

2. review of related research work

2.1 Methods of Data Aggregation

This section covers detailed literature review of data collection and aggregation and the impact of data aggregation on wireless sensor networks with more focus on the delay problem caused by the process of data aggregation on different architectures of wireless sensor networks. The relationship between delay and energy with respect to data aggregation has been discussed in ( $\mathrm{Li}$ et al 2010). Their research has shown that aggregation helps to eliminate the data redundancy hence improving the energy consumption but at the same time increases delays. The paper identifies the two basic methods for data aggregations; non-aggregation and full aggregation. The non-aggregation method induces no delay while full aggregation causes some delays, is favourable for energy saving. However, the authors recognize that some applications do require both energy saving and minimum delay.

minimum data delay. For this reason a partial data aggregation method is suggested.

The two basic methods of data aggregation as well as the partial data aggregation are described below:

2.1.1. Non-aggregation method

This method is one of the timely transmit technologies. Data will be transmitted to the sink (the destination), node by node. For example, suppose there are 5 nodes from $\mathrm{n} 1, \ldots ., \mathrm{n} 5$ as shown in Fig 1 (adopted from ( $\mathrm{Li}$ et al 2010)) and the channels linking the nodes are using CSMA/CD. In CSMA/CD model of communication, Event E51 occurs at n5 then Data D51 
will be transferred from $n 5$ to the adjacent lower node n4, after that event E41 is observed on n4 and E31 on node three. In this case node 3 has to wait until the data transmission is finished for node 4 to avoid data collision.

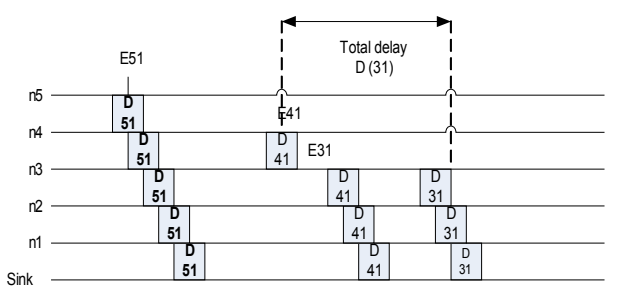

Figure 1: Non-Aggregation method

2.1.2. Full aggregation method

Here the main idea is when a node observes an event first it checks whether there are received data from upper nodes or not. If so, then it aggregates all the data according to an aggregation factor, which has different values and detects how much data can be aggregated through a channel. Then the node transmits the aggregated data to the adjacent lower node. If it does not receive any data then it transmits the data generated by itself to the adjacent lower node. For example in Fig 2 the data from node $\mathrm{n} 5$ will not be transmitted further until E41 occurs at node 4 . After event occurs on node 4 data from node 5 will be aggregated together with the data from node 4 and transmitted to node 3 .

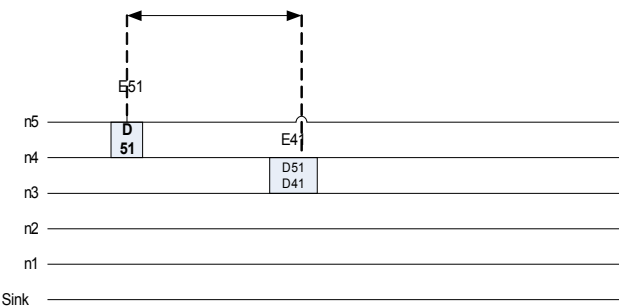

Figure 2: Full Aggregation method

\subsubsection{Partial data aggregation method}

In full aggregation the data transmission of an event is dependent on the happening of new event on the node adjacent to it and hence causing some long delays. The proposed partial aggregation method overcomes this problem by allowing some data to be aggregated and some are not. Therefore the waiting time of one event is not dependent on the occurrence of another event thus reducing the waiting time. In the Fig 3 the event E31 will not wait for event E32 once it notices any event in short time it will transfer the data to the next node as shown in Fig 3.

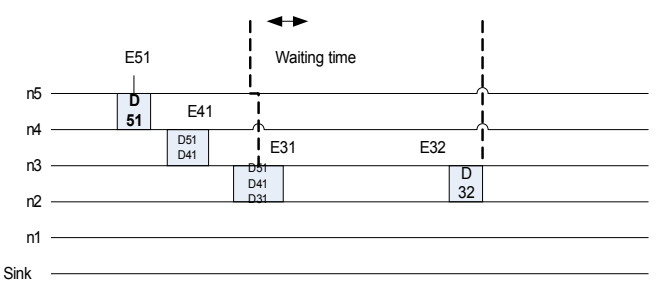

Figure 3: Partial aggregation method

The delay problem in data aggregation in sensor networks is studied by researchers in a variety of situations such as tree topology network, clusters, mobile sink based networks and event driven communication. Below we discuss these scenarios and summarize findings of researchers in these scenarios with respect to data aggregation.

2.2 Types of wireless sensor networks according to data collection methods

Wireless sensor networks can be divided into three different types according to the method used for data collection (Jurdak 2007). These types are:

\subsubsection{Time-driven sensor networks}

In time driven sensor networks, nodes collect and report data from the physical environment periodically. One of the best examples of time-driven sensor networks are those sensor nodes used under water to monitor physical indicators such as temperature, wave direction, and pressure. Fig 4 shows the process of periodic data transfer from source nodes to the base station. Small circles (coloured red) represent sensor nodes deployed in the field and the bigger circle (coloured blue) represents a base station. Every node keeps sensing the environment and sends data to the base station on a regular basis or periodically. 


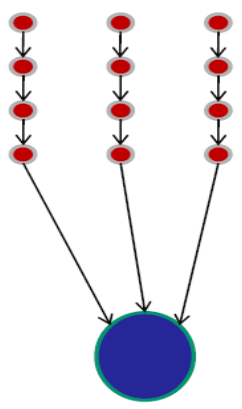

Figure 4: Periodic data transfer in data-driven sensor networks

2.2.2. Event-driven sensor networks

Unlike time-driven WSN, nodes in event-driven sensor networks do not send data periodically. Instead, nodes adjust their data reporting behaviour based on the sporadic happening of certain events. This type of networks is used in situations like monitoring forest fires, intrusions where information from any triggered node is received by all nodes. So low latency or delay is key for event-driven sensor networks. Fig 5 explains this type of sensor network. The black dot within the region identified with a circle represents the exact location of event occurrence. Only those nodes in the vicinity of the event that will sense the event collect and transfer the information to the base station.

Figure 5: Event-driven data transfer

\subsubsection{Demand-driven sensor networks}

This approach is also known as query-driven sensor networks. In this type of networks network entities such as an end user or software component, are enabled to query the nodes for sensed data. This approach is used in cases when the periodic data collection may not work properly because of the overhead of transmitting redundant data. The protocol of this approach involves two steps, (i) The sink or the base station sends a request to sensor nodes to transmit the data as shown in Fig 6.a and (ii) depending upon this request the sensor nodes transmit the data back to the sink as illustrated in Fig 6.b.

Figure 6: Query-based data transfer

2.3. Topologies of wireless sensor networks

Following is an account of different topologies of wireless sensor networks and how each one deals with the issues of delay and energy consumption.

\subsubsection{Tree topology-based sensor network:}

As argued in (Krishnamachari et al 2002), the main difference between Data-centric protocol and Addresscentric protocol is how data is sent from sensor nodes to sinks. In Address-centric Protocol, also known as endto-end routing, each source sends data to the sink independently along the shortest path. On the other hand in Data-centric Protocol, also known as in-network data aggregation, data sent to the sink could be aggregated by intermediate nodes using min, max, duplicate suppression functions. In in-network data aggregation in treebased structure, data aggregation may happen at various nodes consequently improving the energy consumption of the entire network (Joo et al 2010). The longer an intermediate node holds data before forwarding it the better the throughput efficiency because of greater aggregation. However, this could result in longer end-to-end delay. In their research Joo et al (2010) propose to combine in-network data aggregation with scheduling where nodes can use a single frequency by taking turns in equal time durations using one channel in order to minimize end-to-end delays.

\subsubsection{Cluster topology-based sensor networks:}

Clustering scheme has been proved to be an efficient way of data aggregation where nodes are clustered together and data forwarded to cluster head is aggregated before sending it further (Min et al 2012). The cluster based scheme is organized into two categories: homogeneous and heterogeneous. In a cluster based sensor network the nodes are divided into different clusters where each cluster has its leading node called the cluster head. All the nodes send data to the cluster head that further send that data to sink node or to the other clusters. In homogeneous scheme all the nodes have the same energy and the probability to be a cluster head depends on the remaining energy of a node. This means the node that is left with more energy than the other nodes will become automatically the cluster head. And therefore there is no need of election process of cluster head. In heterogeneous cluster networks there are two different types of nodes; Type-1 and Type-2. Type-1 
nodes are small, low cost with low energy consumption. They are simply designed for basic sensing. Type-2 nodes also called cluster heads are responsible for aggregating the intra-cluster data coming from the Type -1 nodes. Type-2 nodes have more powerful energy sources than that of Type-1. Since we have 2 different types of nodes with different energy sources, increasing the lifetime of such heterogeneous cluster network proved to be more challenging for designers and researchers.

3. THE PROPOSED METHODOLOGY

Our proposed methodology is an improvement of the scheme introduced in Tree-based Clustering for Energy Efficient Wireless sensor Network (Kung et al 2010). The simulation results show that the scheme has effectively reduced and balanced the energy consumption among the nodes. Our idea is to combine the treebased Clustering with partial aggregation as discussed in ( $\mathrm{Li}$ et al 2010). The new method that we are developing is to combine the concept and attributes of tree with clustering by forming many clusters and inside each cluster, nodes are distributed in a form of tree structure. Each cluster will have many cluster heads according to their position from the sources. We also assume that each cluster head acts as an aggregator. We will also take advantage of partial aggregation where needed. The idea is to divide the aggregation process among different cluster heads. For example as shown in Fig 7, node $\mathrm{n} 1$ and node $\mathrm{n} 2$ will send the data to aggregator Ag1 where data will be aggregated and sent further to the Ag2. However data might also be received from node $\mathrm{n} 3$ and node $\mathrm{n} 4$. If $\mathrm{n} 3$ and $\mathrm{n} 4$ have to wait for a long time for the arrival of data from Ag1, a possible considerable delay can be experienced. Therefore by setting a time deadline data which, is not received by Ag1 will have to be sent to the main aggregator directly. With such arrangement, it becomes apparent that one of the central functions of the main aggregator is to reduce the duplicate data coming from different clusters that will help increasing the lifetime of the sensor nodes and at the same time the use of partial aggregation will help reducing delays.

Figure 7: Tree-based clustering with partial aggregation

4. CONCLUSION

Event-driven sensor networks are very important and commonly used as a data collection structures in many applications of wireless sensor networks. The two main concepts domineering the efficacy of these types of networks are the frequency of data collection $(f d c)$ and the amount of energy consumption $(e c)$.

By utilizing the properties of the structures of the wireless sensor networks; the tree and cluster together with the exploiting of partial aggregation, delays in data collection can be minimized while the lifetime of energy sources can be maximized. Research work is still underway to calculate the optimum values of these two parameters $(f d c$ and $e c$ ) of the suggested model that will ensure energy is preserved without compromising the frequency of data collection.

\section{References}

Joo, C., Choi, J. and Shroff, N.B. (2010), Delay Performance of Scheduling with Data Aggregation

\author{
Diego, USA
}

Jurdak, R. (2007) Wireless Ad Hoc and Sensor Networks: A Cross-Layer Design Perspective. Springer

Kim, K.T., Lyu, C.H., Moon, S.S. and Youn, Efficient Wireless Sensor Networks.

H.Y. (2010) Tree-Based Clustering (TBC) for Energy Networking and Applications Workshops

IEEE 24th International Conference on Advanced Information (WAINA), Pages 680- 685

Krishnamachari, B., Estrin, D. and Wicker, S. (2002), Impact Of Data Aggregation In Wireless Sensor Networks. Proceedings of the 22nd International Conference on Distributed Computing Systems, 2002, Vienna, Austria

Li, W., Okamura, D., Bandai, M. and Watanbe, T. $\quad$ (2010), Tradeoff Between Delay And Energy Consumption Of Partial Data Aggregation In Wireless Sensor Networks. Proceedings of the $24^{\text {th }}$

IEEE International Conference on Advanced Information Networking and Applications. 2010, Perth, Australia

Min, Y., Chuang, L. and Yuan, T. (2012) Energy And Delay Minimization In Cluster-Based

Sensor Networks. IEEE 2012. Besançon, France
Wireless

Computing, 